\title{
O Conceito de Operação Estabelecedora na Análise do Comportamento ${ }^{1}$
}

\author{
Caio F. Miguel ${ }^{2}$ \\ Western Michigan University
}

\begin{abstract}
RESUMO - Em termos gerais, operações estabelecedoras podem ser definidas como eventos ambientais que alteram a efetividade reforçadora de um estímulo, assim como evocam todo comportamento que, no passado, foi seguido por tal estímulo. O conceito parece descrever, em termos comportamentais, o que é usualmente chamado de motivação. O presente artigo pretende apresentar de uma forma didática o desenvolvimento teórico do conceito e seu status na Análise Experimental e Aplicada do Comportamento.
\end{abstract}

Palavras-chave: operações estabelecedoras; motivação; análise do comportamento; comportamentalismo.

\section{The Concept of Establishing Operation in Behavior Analysis}

\begin{abstract}
In general terms, establishing operations can be defined as environmental events that alter the reinforcing effectiveness of a stimulus, as well as evoke all behavior that, in the past, has been followed by such a stimulus. The concept seems to describe, in behavioral terms, what is usually called motivation. The present paper is an attempt to didactically present the theoretical development of the concept and discuss its current status in the Experimental Analysis of Behavior and Applied Behavior Analysis.
\end{abstract}

Key words: establishing operations; motivation; behavior analysis; behaviorism.

Em linguagem comum, muitos dos problemas ditos psicológicos podem ser divididos em problemas de aprendizagem ou de motivação. É comum falarmos na diferença entre o "saber" e o "querer" quando nos referimos a aprendizagem e motivação, respectivamente. Na análise do comportamento, problemas de motivação vêm sendo explicados a partir do conceito de "reforçamento" (Michael, 1993a). O problema do "querer" (não querer fazer algo) muitas vezes tem suas raízes na falta de reforçamento disponível para que o comportamento ocorra. Entretanto, o conceito de reforçamento não dá conta de explicar todos os problemas relacionados à motivação. Muitas vezes o problema não está na falta de consequiências para o comportamento, mas na ineficácia de tais conseqüências.

O conceito de operações estabelecedoras, primeiramente introduzido na literatura por Keller e Schoenfeld (1950/ 1996) e, mais tarde, reintroduzido por Michael (1982, 1993a) pretende dar conta de explicações que incluem variáveis ditas motivacionais. O objetivo deste texto é discutir o desenvolvimento teórico de tal conceito e seu estágio atual na Análise do Comportamento, dando ênfase às demonstrações experimentais.

1 Agradeço aos professores Kester Carrara e Maria Amélia Matos pelos comentários e sugestões; e ao Prof. Jack Michael pela inestimável orientação.

2 Endereço: Department of Psychology, Western Michigan University, Kalamazoo, Michigan 49008, EUA. Email: caio.miguel@wmich.edu

\section{O Conceito de Drive}

Entre as variáveis das quais o comportamento é função, incluem-se aquelas que se pressupõe estarem operando durante o fortalecimento de uma resposta: as variáveis motivacionais. Operações como as de privação de alimento são pré-requisito para que o alimento possa, como conseqüência de uma resposta, adquirir função reforçadora. É também fato sabido que determinado organismo não se alimenta continuamente, mas sim em determinadas situações e em quantidades específicas, ou seja, quando ele está "faminto". Parece que a privação de alimento altera o valor do alimento tornando-o um estímulo reforçador ${ }^{3}$, assim como evoca comportamentos que no passado produziram alimento.

Skinner (1938/1991) e Keller e Schoenfeld (1950/1996) sugerem o termo Drive ${ }^{4}$ como capaz de definir, ou simplesmente identificar operações como as de privação e saciação. De acordo com Skinner (1938/1991), cada drive possui suas próprias operações definidoras (p. 358). No caso

3 Estímulo reforçador será usado daqui por diante para descrever uma conseqüência cuja apresentação imediata após determinada resposta aumenta a probabilidade de ocorrência futura de respostas da mesma classe. Na verdade não é o estímulo que funciona como reforçador mas a mudança entre uma situação na qual o estímulo não estava presente para uma situação na qual ele passa a estar presente. Para uma maior discussão a respeito do conceito de mudança de estímulo vide Michael (1993a).

4 O termo Drive não será traduzido por impulso para não ser confundido com termos derivados de outras abordagens da Psicologia. 
da "fome" (como um Drive) a operação definidora seria a privação de alimento. Keller e Schoenfeld (1950/1996) propõem o termo "operações estabelecedoras de drive" para descrever essas operações. De acordo com estes autores, operações estabelecedoras de um drive, teriam como efeito: 1) a mudança momentânea de um grupo de respostas (evocar a ocorrência das respostas) e 2) tornar o reforçamento possível (estabelecer a conseqüência como reforçadora).

\section{Função Motivacional}

Michael, em 1982, apresentou uma proposta de reformulação taxonômica no que diz respeito à inclusão ou diferenciação entre a função motivacional e discriminativa do estímulo. De acordo com o autor, a mudança de S-delta para $S^{D}$ é uma mudança que - "dada a efetividade momentânea de algum evento como reforçador" (função motivacional) - aumenta a frequiência de uma resposta porque esta mudança foi correlacionada com um aumento na disponibilidade de um evento reforçador (função discriminativa). $\mathrm{O}$ autor afirma que em muitos casos leva-se em conta o controle do comportamento por um estímulo discriminativo, deixando de lado variáveis como privação e saciação que estariam em operação. Estas variáveis (motivacionais) podem estar afetando diretamente o comportamento de duas maneiras: 1) aumentando a efetividade do reforçador, que caso siga qualquer novo comportamento, passaria a selecioná-lo; e 2) aumentando a freqüência de todas as respostas que foram fortalecidas pelo reforçador em questão. Michael (1982) descreve o primeiro efeito como estabelecedor do reforço e o segundo efeito como evocativo (semelhante ao exercido pelo $\mathrm{S}^{\mathrm{D}}$ ).

Malott, Malott e Trojan (2000) também definem variáveis de motivação a partir desses dois efeitos. Para estes autores, uma operação como a de privação de água afetaria o organismo 1) durante o treino de uma resposta e 2) durante o desempenho da mesma. Quando, por exemplo, o pressionar a barra produz água como consequiência, esta só funcionaria como um reforçador durante o fortalecimento operante (treino) caso o organismo estivesse privado. Da mesma forma, após o treino, o organismo só pressionaria a barra caso estivesse privado de água. $\mathrm{O}$ grande problema de se definir o conceito a partir destes dois efeitos é que o efeito estabelecedor da operação estabelecedora não pode ser observado diretamente, mas somente inferido a partir do efeito evocativo (Cherpas, 1993).

Até o momento, o que os autores parecem tentar ressaltar é que o valor reforçador de uma conseqüência depende diretamente da operação estabelecedora, ou seja, a menos que a operação estabelecedora esteja em efeito a relação entre os três termos da contingência, não é efetiva.

Michael (1982) revisita Keller e Schoenfeld (1950/1996) com uma única diferença: o conceito de Drive. Para estes últimos, o conceito de operações estabelecedoras sempre esteve presente como parte de um conceito maior - o conceito de drive. Michael, preferiu abandonar o termo drive, utilizando apenas o termo operações estabelecedoras ("OES"), deixando clara a relação ambiente-organismo, na tentativa de evitar associações com propostas mentalistas para a motivação. Vale a pena ressaltar que tanto Skinner (1938/1991) quanto Keller e Schoenfeld (1950/1996) afirmaram que o termo drive é apenas um instrumento conceitual e que poderia ser analisado e refinado.

De acordo com Michael (1982, 1988, 1993a), eventos como os de privação/saciação e estimulação aversiva parecem possuir características em comum. Eles momentaneamente alteram a) a efetividade reforçadora de outros eventos e b) a frequiência de ocorrência de todo o comportamento que foi reforçado por esses eventos. Eventos que possuem estas duas características passaram a ser categorizados pelo autor como operações estabelecedoras. Em sua definição mais completa Michael (1993a, 1993b) inclui quatro efeitos distintos comuns às operações estabelecedoras:

1. Efeito estabelecedor de reforçamento: uma operação estabelecedora, momentaneamente, altera a efetividade reforçadora (ou punidora) de um estímulo. No caso, privação de água (como uma operação estabelecedora) aumentaria a efetividade de água como reforçador.

2. Efeito evocativo/supressivo da operação estabelecedora: imediatamente evoca (ou suprime) comportamentos que no passado produziram consequiências cuja efetividade tenha sido alterada em (1). Assim, privação de água, como um evento antecedente (OE) evoca qualquer comportamento que tenha sido seguido pela apresentação de água.

3. Efeito evocativo/supressivo do $S^{D}$ : aumenta a efetividade evocativa (ou supressiva) de todos os estímulos discriminativos $\left(\mathrm{S}^{\mathrm{D}} \mathrm{s}\right)$ que tenham sido correlacionados com o estímulo reforçador ou punidor em (1). Em um experimento de discriminação simples, uma luz na caixa operante é correlacionada com a disponibilidade diferencial de reforçamento. A efetividade evocativa da luz depende do grau de privação do sujeito $(\mathrm{OE})$.

4. Efeito sobre o reforçamento/punição condicionada: aumenta/diminui a efetividade reforçadora/punidora de qualquer reforço/punidor (condicionado) cuja efetividade depende do reforçador/punidor em (1). Dada a seguinte cadeia comportamental

$$
\text { passa pela argola } \rightarrow \text { som } \rightarrow \text { pressiona a barra } \rightarrow \text { água }
$$

Privação de água (OE) aumenta a efetividade do som como uma forma de reforçamento condicionado e aumenta a freqüência de passar pela argola porque o som foi consistentemente relacionado com a apresentação de água (para a resposta de pressão à barra).

Analisando o exemplo anterior, temos que a privação (OE) 1) aumenta a efetividade reforçadora da água; 2) aumenta a freqüência de pressões a barra pois no passado tal comportamento resultou em obtenção de água; 3) aumenta a efetividade evocativa do som; e 4) aumenta a efetividade do som como reforçador condicionado - assim o som passaria a aumentar a freqüência de qualquer comportamento que o tenha precedido (no caso, passar pela argola). 


\section{O Estímulo Estabelecedor}

A contribuição de Michael (1982) parece ir além de uma redefinição conceitual, quando discute as operações estabelecedoras que alteram a efetividade de reforçadores condicionados. Da mesma forma que outros autores (Skinner, 1953/ 1988; Millenson, 1967/1975), Michael aponta a efetividade do reforçador condicionado como função do valor do reforçador primário com o qual ele foi pareado (efeito 4). Entretanto, Michael (1982) ressalta uma situação na qual uma mudança de estímulo estabelece outra condição como reforçadora (reforçador condicionado) sem a alteração da efetividade do reforçador primário relevante. Para esta relação ele propõe o termo estímulo estabelecedor $\left(\mathrm{S}^{\mathrm{E}}\right)$.

Podemos observar o estímulo estabelecedor em operação numa situação na qual um estímulo $S^{1}$ estivesse funcionando como um estímulo discriminativo $\left(S^{\mathrm{D}}\right)$ para uma resposta $\mathrm{R}^{1}$, que não poderia ser emitida ou reforçada até que outro estímulo $\mathrm{S}^{2}$ estivesse presente. Este segundo estímulo tornar-se-ia, portanto, efetivo como reforçador condicionado e o comportamento $\left(\mathrm{R}^{2}\right)$ que produz $\mathrm{S}^{2}$ seria evocado. $S^{1}$ funcionaria, desta forma, como um estímulo discriminativo para $\mathrm{R}^{1}$ e, ao mesmo tempo, como um estímulo estabelecedor para $\mathrm{R}^{2}$. $\mathrm{O}$ exemplo mais clássico de Michael (1982), descreve a seguinte proposta de delineamento experimental para mostrar esta função de estímulo em animais:

Consideremos um macaco privado de comida em uma câmara com uma corrente no teto e uma barra retrátil. Puxar a corrente move a barra para dentro da câmara. Pressionar a barra não tem efeito ao menos que uma luz na parede esteja acesa, momento esse em que a pressão à barra tem como conseqüência uma pelota de comida. Para prevenir que o puxar da corrente funcione como um componente padrão preparatório de uma seqüência comportamental (...) nós poderíamos deixar a corrente na posição puxada ou poderíamos permitir que cada puxada na corrente produzisse a presença da barra por um período limitado, digamos, 5 segundos (p. 153).

Para Michael (1982), na situação em que a luz estivesse apagada, o macaco não puxaria a corrente, mesmo que produzisse a barra (reforço condicionado) e na situação na qual a luz estivesse acesa, o macaco puxaria a corrente, pressionaria a barra e comeria a pelota de alimento. $\mathrm{O}$ autor considera que a luz $\left(S^{1}\right)$ estaria funcionando como um $S^{D}$ que evocaria o comportamento de pressionar a barra $\left(\mathrm{R}^{1}\right)$. Porém esta resposta (pressionar a barra) não poderia ocorrer ou não poderia ser reforçada sem a presença da barra $\left(\mathrm{S}^{2}\right)$. Assim, a luz $\left(\mathrm{S}^{1}\right)$ funcionaria, também, como um $\mathrm{S}^{\mathrm{E}}$, estabelecendo a visão da barra $\left(\mathrm{S}^{2}\right)$ como uma forma de reforçamento condicionado e ao mesmo tempo evocando o comportamento de puxar a corrente $\left(\mathrm{R}^{2}\right)$, que anteriormente produziu a barra $\left(\mathrm{S}^{2}\right)$. A luz $\left(\mathrm{S}^{1}\right)$, com relação à $\mathrm{R} 2$, estaria funcionando como um $S^{\mathrm{E}}$ e não como um $\mathrm{S}^{\mathrm{D}}$, porque ela (a luz) não esteve correlacionada com uma maior disponibilidade da barra (reforçador condicionado para o puxar a corrente), mas sim com a maior efetividade da barra como uma forma de reforçamento.

Em um exemplo mais simples, imaginemos o rato na caixa de Skinner. Na presença de uma luz, o rato pressiona a barra e recebe água. $\mathrm{Na}$ ausência da luz, o rato pressiona a mesma barra e nada ocorre. Neste caso, privação de água funcionaria como uma operação estabelecedora que alteraria o valor da água como reforçador. A luz funcionaria como um $S^{\mathrm{D}}$, que passa a evocar respostas de pressão a barra dada a maior disponibilidade de água em sua presença. $\mathrm{Na}$ ausência da luz (S delta), o rato não pressionaria a barra já que a água não esteve disponível nesta condição. Luz acesa funcionaria também como uma operação estabelecedora, alterando o valor da barra como uma forma de reforçamento condicionado (a presença da barra é condição necessária para que a resposta de pressão ocorra). Quando a luz esteve acesa, a barra foi pareada com a água. $\mathrm{O}$ valor da barra como reforço condicionado (para respostas de olhar ou aproximar-se da barra) depende da presença da luz. Assim, luz acesa, como uma OE evocaria comportamentos de observação da barra e, como um $\mathrm{S}^{\mathrm{D}}$ evocaria respostas de pressão na mesma. Mais tarde Michael (1993a) passou a se referir a esta relação como operação estabelecedora condicionada transitiva, como veremos a seguir.

\section{Operações Estabelecedoras Condicionadas}

Em 1988, Michael propõe uma divisão entre operações estabelecedoras incondicionadas (OEIs) ${ }^{5}$ e operações estabelecedoras condicionadas (OECs) $)^{6}$. De acordo com o autor, uma operação estabelecedora incondicionada envolveria operações que têm efeitos de estabelecimento sobre os reforçadores, independente de qualquer tipo de aprendiza$\mathrm{gem}^{7}$. Tais operações como a privação, estimulação aversiva, ingestão de sal, mudança de temperatura, fariam parte dessa categoria.

Um outro grupo, o das operações estabelecedoras condicionadas, incluiria aquelas operações cujo efeito de estabelecimento do reforçador é aprendido. Ou seja, operações cuja alteração no valor de um evento como reforçador dependem da história individual do organismo. Vale a pena resssaltar que a distinção entre operações estabelecedoras incondicionadas (OEIs) e condicionadas (OECs) depende somente do efeito estabelecedor (caso seja inato ou aprendido, respectivamente) e não do efeito evocativo. No caso, privação de água altera a efetividade de água (reforçador incondicionado) independente de qualquer aprendizagem, já a função evocativa de qualquer operação estabelecedora, seja ela condicionada ou incondicionada, depende da apredizagem, já que só pode evocar comportamento operante.

De acordo com Michael (1988) eventos ambientais como os estímulos sinalizadores de uma contingência de esquiva

5 Unconditioned Establishing Operations

6 Conditioned Establishing Operations

7 Vale a pena ressaltar que tanto Keller e Schoenfeld (1950/1996), quanto Millenson (1967/1975) fazem referência aos "efeitos sobre o valor do reforçador primário" deste tipo de operação. 
também poderiam ser categorizados como operações estabelecedoras. Segundo Michael, o estímulo sinalizador, por ter sido pareado com a situação aversiva (ex. choque), torna sua presença suficiente para que respostas que o evitem sejam evocadas. Para ser categorizado como um $\mathrm{S}^{\mathrm{D}} \mathrm{o}$ estímulo sinalizador deveria possuir uma situação análoga ao S-delta. Supondo que o reforçador para a resposta de esquiva seja a eliminação do estímulo sinalizador (consequiência imediata), sua ausência impossibilita que o reforçador negativo ocorra. Ou seja, a efetividade do reforçador negativo depende diretamente da presença do estímulo aversivo condicionado. $\mathrm{O}$ mesmo ocorre na situação de fuga, a ausência do choque só é reforçadora caso o choque esteja presente. $\mathrm{O}$ choque não pode funcionar como um $\mathrm{S}^{\mathrm{D}}$ porque na sua ausência (suposto $S$-delta) a remoção do choque não é reforçadora. Veja que quando nos referimos à discriminação, a conseqüência é efetiva tanto na condição de $\mathrm{S}^{\mathrm{D}}$ quanto na de S-delta, ou seja o rato na caixa de Skinner está privado de água nas duas condições, de modo que a única diferença é que em S-delta ele não recebe a água. Para o choque funcionar como um $\mathrm{S}^{\mathrm{D}}$, a remoção do choque deveria funcionar como reforçadora também em S-delta. Entretanto, quando o choque não está presente sua remoção é logicamente impossível. Assim tanto o estímulo aversivo, quanto o estímulo sinalizador de uma contingência de esquiva funcionam como operações estabelecedoras e não $S^{D}$ s. Por possuir a função de estabelecer sua própria ausência como reforçadora Michael (1993a) chamou esta relação (somente a apresentação do estímulo aversivo condicionado) de operação estabelecedora (condicionada) $r e$ flexiva ${ }^{8}(O E C-R)$.

Um outro tipo de relação também pode ser incluída no conjunto das OECs. Seriam as situações nas quais eventos neutros, quando pareados com operações estabelecedoras incondicionadas (OEIs) adquiririam as mesmas funções. Um exemplo seria o pareamento de uma luz (estímulo neutro) com redução de temperatura (OEI). Seria esperado que a luz adquirisse não só funções respondentes (eliciadoras) ou aversivas/reforçadoras, mas também estabelecedoras. Sua presença deveria estabelecer o aumento de temperatura como uma forma de reforçamento. Entretanto, de acordo com Mineka (1975), a descrição deste fenômeno é puramente teórica e experimentos na área, são escassos e inconclusivos $^{9}$ (vide Knip, 1991). Michael (1993a) chamou este conjunto de relações de OEC Substitutas ${ }^{10}$ (OEC-S).

O terceiro grupo de Operações Estabelecedoras, as Transitivas $^{11}$ (OEC-T), envolve o que anteriormente foi chamado de Estímulo Estabelecedor (exemplo do macaco). A discussão a respeito deste tipo de OEC foi avançando a cada

8 Reflexive $\mathrm{CEO}$

9 Há uma especulação evolucionária que sustenta o argumento do porquê não seria possível observarmos os efeitos da OEC-S (Mineka, 1975; Michael, 1999). De acordo com a definição de OEC-S características do ambiente onde determinado organismo esteve faminto adquiriria efeitos de OEC através de pareamento (privação $\leftarrow \rightarrow$ características do ambiente). No futuro, estas características do ambiente funcionari- trabalho publicado pelo autor (Michael 1982, 1988, 1993a, 1993b, 2000).

Em 1982, como visto anteriormente, Michael define $S^{\mathrm{E}}$ como um estímulo que teria a função de modificar a efetividade reforçadora condicionada de um estímulo. Em 1988, Michael abandona o termo $S^{\mathrm{E}}$ e introduz o termo Operação Estabelecedora Condicionada de Resposta Bloqueada ${ }^{12}$, tentando especificar uma relação na qual determinado estímulo poderia funcionar como $\mathrm{S}^{\mathrm{D}}$ para uma resposta, que de certa forma estaria bloqueada ou não poderia ocorrer, até que outro objeto ou evento se tornasse presente. No caso da discriminação simples, quando a luz está acesa, o rato só consegue pressionar a barra para obter água se a barra estiver presente. Assim, a luz funciona como OEC de resposta bloqueada evocando respostas que produzem a presença da barra como consequiência.

É só em 1993a, quando divide as Operações Estabelecedoras Condicionadas em três grupos, que Michael passa a se referir a estas relações como OECs Transitivas.

$\mathrm{O}$ autor parece, em todo seu trabalho, enfatizar que este tipo de OEC está muito presente em situações do dia-a-dia. Em outro exemplo com humanos (Michael, 1982;1993a) descreve o comportamento de um eletricista que frente a um parafuso precisa, para desparafusá-lo, de uma chavede-fenda. A visão do parafuso evocaria o comportamento verbal de solicitar a chave de fenda a um ajudante, não como um $S^{\text {D }}$, mas sim como uma OEC, por não estar correlacionada diferencialmente com a obtenção de chaves-de-fenda, mas por torná-la, nesta situação, um reforçador condicionado efetivo. Para que o parafuso seja categorizado como um $\mathrm{S}^{\mathrm{D}}$, a chave-de-fenda precisaria 1) estar mais disponível na presença do parafuso do que em sua ausência e 2) funcionar sempre como um reforçador condicionado. Entretanto, a chave-de-fenda estaria igualmente disponível na presença e ausência do parafuso. A probabilidade que o eletricista tem de obter a chave de fenda (caso peça a seu assistente) é a mesma independente da visão do parafuso. Entretanto, é só na presença do parafuso que a chave-de-fenda funciona como um reforçador. Já a pessoa para a qual o eletricista pediu a chave de fenda (o assistente) funcionaria como um $\mathrm{S}^{\mathrm{D}}$ para a emissão do pedido, pois se supõe ter existido maior probabilidade de reforçamento por pedir a chave-de-fenda na presença do assistente, que em sua ausência.

A noção de Operação Estabelecedora Condicionada Transitiva proposta por Jack Michael, vem sendo discutida e algu-

am como OEC-S que alterariam a efetividade da comida como reforçador, evocando qualquer comportamento que foi seguido por comida no passado, independente de uma privação de comida. Tal efeito poderia ser prejudicial ao organismo que biologicamente não necessita de comida. O oposto poderia ser ainda mais prejudicial. Eventos ambientais pareados com saciação passariam a exercer a função de diminuir a efetividade de comida como reforçador, independentemente das necessidades biológicas do organismo.

10 Surrogate CEO

11 Transitive CEO: o termo, segundo Michael (1993a) foi escolhido como uma analogia à um verbo transitivo que necessita de um objeto direto.

12 Blocked-Response CEO 
mas vezes criticada por diversos autores (Catania, 1993; Da Cunha, 1995; Hesse, 1993; McDevitt \& Fantino, 1993; Schlinger, 1993; Sundberg, 1993). A principal crítica ao conceito de OEC é elaborada por McDevitt e Fantino (1993) e envolve a problemática da distinção entre $\mathrm{S}^{\mathrm{D}}$ e OEC. Segundo os autores, os exemplos dados por Michael (1993a) esclarecem os efeitos estabelecedores do reforçador, entretanto são confusos no que se refere ao controle de estímulo responsável pela emissão da resposta (função evocativa). Aquilo que Michael (1993a) chamou de OEC Transitiva parece funcionar como um estímulo, que juntamente com o suposto $S^{D}$ estaria sinalizando a disponibilidade do reforço.

McDevitt e Fantino (1993) ao analisarem o exemplo do eletricista, descrito anteriormente, interpretam o controle exercido pela OEC-T como discriminativo. A visão do parafuso controlaria diretamente o comportamento do eletricista de pedir pela chave-de-fenda como um $\mathrm{S}^{\mathrm{D}}$. O pedido produz, na presença do parafuso, os eventos conjuntos: chave de fenda e (eventualmente) a remoção do parafuso (e mais remotamente, produz todas as conseqüências relacionadas a conclusão dessa tarefa). A interpretação proposta pelos autores é a de uma unidade funcional em que o parafuso funciona como um $S^{D}$ para toda a seqüência de respostas em questão. Entretanto ao considerarmos o pedido da chave de fenda como sendo controlado por eventos conjuntos, que agem como uma forma de reforçamento, estamos negligenciando o controle imediato do reforçador condicionado (obtenção da chave de fenda) sobre a resposta de pedir e supondo que esse comportamento pode ser diretamente reforçado por conseqüências remotas. Tal suposição nos levaria a questionar a necessidade da proximidade temporal para que o condicionamento operante ocorra (Malott, 1984; Michael, 1986).

\section{Múltipla Função}

Como discutido em alguns dos exemplos apresentados, um mesmo estímulo pode adquirir diferentes funções comportamentais, em relação à diferentes respostas. Um estímulo pode também exercer múltiplas funções estabelecedoras. A situação de esquiva descreve bem como o estímulo usualmente chamado de sinalizador pode adquirir diversas funções sobre diferentes respostas.

Suponha um estímulo S1 que foi sistematicamente precedido por um aumento "desconfortável" de temperatura (OEI). Suponha que S1 poderia ser eliminado antes que a temperatura aumentasse, nesse caso, a eliminação de S1 faria com que a temperatura permanecesse "confortável" até que S1 ocorresse novamente. Nesse caso, S1 como um estímulo sinalizador, exerceria diversas funções: 1) eliciaria, como um estímulo condicionado, respostas relacionadas a um aumento de temperatura; 2) funcionaria como punidor diminuindo a frequiência de qualquer resposta que o precedesse; 3) evocaria, como uma OEC-S (dado o pareamento entre S1 e o aumento de temperatura), todo comportamento que no passado foi seguido por um decréscimo de temperatura; 4) evocaria, como uma OEC-R, todo comporta- mento que foi seguido pelo término de $\mathrm{S} 1$ (seu próprio término) e; 5) evocaria, como uma OEC-T, todo comportamento precorrente à emissão da resposta que elimina $\mathrm{S} 1$, por exemplo respostas visuais para o operandum que produz a remoção de $\mathrm{S} 1$.

O conceito de operação estabelecedora, como instrumento conceitual, parece contribuir para uma análise mais minuciosa das variáveis das quais o comportamento é função. Entretanto, é importante também que tal conceito possa ser reproduzido em situação experimental.

\section{Demonstrações Experimentais $^{13}$}

Preocupados em demonstrar experimentalmente aquilo que Michael (1982) chamou de $\mathrm{S}^{\mathrm{E}}$, McPherson e Osborne $(1986,1988)$ conduziram dois experimentos com animais. No primeiro deles, quatro pombos foram colocados na seguinte situação experimental: No início de cada tentativa, um disco verde (direita) era iluminado. Ao bicarem esse disco iluminava-se um outro disco branco no centro. A primeira bicada no disco branco, no centro, produziria alimento caso um disco de vermelho, à esquerda, estivesse iluminado. A luz vermelha era apresentada aos sujeitos num esquema de tempo variável ou randômico (60s). Na ausência da luz vermelha, bicar a chave branca não produziria alimento como conseqüência. As tentativas se encerravam após acesso ao alimento. Neste procedimento, a iluminação do disco branco no centro deveria funcionar como um reforçador condicionado para a resposta de bicar o disco verde à direita. Além disso, a iluminação do disco vermelho à esquerda foi considerada um estímulo estabelecedor que alterava a efetividade do disco branco como reforçador condicionado. As bicadas no disco verde à direita que ocorreram após a iluminação do disco vermelho à esquerda foram consideradas como indicativas do controle pelo estímulo estabelecedor. $\mathrm{O}$ disco vermelho foi considerado uma operação estabelecedora que alteraria o valor do disco do centro como uma forma de reforçamento condicionado. Os resultados obtidos pareceram indicar que o controle exercido pelo suposto $S^{\mathrm{E}}$ foi incompleto, ocorrendo após sessenta sessões. Durante as últimas sessões, em $75 \%$ das tentativas, 3 dos 4 pombos bicaram o disco verde à direita somente após a iluminação do vermelho à esquerda. Em sua discussão, McPherson e Osborne (1986) afirmaram que se poderia supor a luz vermelha como parte de um estímulo discriminativo complexo, ou discriminativo condicional (verde + vermelho). Entretanto, o disco vermelho não esteve, em nenhum momento, correlacionado com uma mudança na probabilidade de que uma bicada na luz verde fosse seguida pela iluminação do disco branco ( $\mathrm{S}^{\mathrm{r}}$ condicionado): a probabilidade de iluminação da luz branca, depois da bicada na verde, era a mesma na presença ou na ausência da vermelha, o que deixaria de caracterizar a luz vermelha como

13 Parte desses experimentos foram descritos por Da Cunha (1995) em uma revisão da literatura experimental sobre OEs. 
um $\mathrm{S}^{\mathrm{D}}$ para o comportamento de bicar o disco verde. McPherson e Osborne (1986) não chegaram a conclusões seguras acerca do controle estabelecedor, afirmando que seus dados estão longe da precisão necessária.

No segundo estudo desenvolvido por McPherson e Osborne (1986), a efetividade do disco branco (iluminado), como reforçador condicionado para respostas de bicar no disco verde, foi manipulada através da redução no tempo entre a iluminação do disco branco e a obtenção da comida. Seus resultados apontaram que, quando a redução do atraso era menor os sujeitos respondiam como se o valor do reforçador condicionado (luz branca-centro) dependesse da presença da luz vermelha (esquerda). Com uma redução maior do atraso, a luz branca (centro) pareceu funcionar como um reforçador condicionado independente da luz vermelha (esquerda). Os resultados descritos por McPherson e Osborne (1988) sugerem que a luz vermelha, na situação da menor redução, poderia ter funcionado como um estímulo estabelecedor: a probabilidade da resposta de bicar o disco verde (direita) aumentou, provavelmente devido a uma maior efetividade do disco branco como reforçador condicionado. Estes resultados podem, também, ser interpretados como um simples encadeamento de respostas controlado pela luz vermelha como um $\mathrm{S}^{\mathrm{D}}$.

Alling (1991) propôs um delineamento experimental, também com pombos, que visava corrigir possíveis falhas nos experimentos descritos anteriormente. Seus pombos deveriam pressionar um pedal (FR1) para produzir uma mudança de luz (acima do pedal/de branca para vermelha) que permanecia acesa por $5 \mathrm{~s}$. A bicada a um disco iluminado nesse período (enquanto a luz vermelha do pedal estivesse acesa) produziria comida por 3 segundos, dependendo da condição da luz da câmara experimental. A mudança da cor da luz do pedal funcionaria como reforçamento condicionado e o valor desse reforço dependeria de outra condição ambiental - a luz da câmara experimental. A ausência ou presença de luz na caixa definiam, portanto, a situação de OEC ou não-OEC, situações que supostamente estariam correlacionadas com a efetividade do reforçador condicionado (mudança da luz do pedal). Cada tentativa se iniciava com a condição OEC (VT 60s) e terminava com a obtenção do alimento. $\mathrm{O}$ desempenho esperado seria a pressão no pedal somente quando a condição de OEC estivesse presente. Todos os pombos comportaram-se como esperado em $90 \%$ das tentativas. Para garantir que a resposta de pressionar o pedal estivesse sendo mantida pela mudança de luz (reforçador condicionado), retirou-se essa condição em uma segunda fase do experimento com o objetivo de se observar quebra na cadeia comportamental. A ruptura na cadeia não foi observada, o que, segundo o autor, poderia ter caracterizado a condição de OEC como $\mathrm{S}^{\mathrm{D}}$.

Da Cunha (1993) replicou o procedimento de Alling (1990), alterando a condição de OEC para um estímulo auditivo e adicionando um esquema de Razão Variável (VR6) para produzir a mudança de cor no pedal, com o objetivo de reduzir a possibilidade da cadeia desenvolver-se como uma unidade funcional, que tornaria o reforçador condicionado desnecessário para a primeira resposta. Os resultados obtidos por Da Cunha mostraram que a taxa de respostas de pressão no pedal na presença da suposta OEC foi maior que na condição de não-OEC. Por outro lado, seus dados, analisados, a partir de porcentagem de tentativas sem erro, não mostram diferenças significativas no desempenho de seus sujeitos.

Hixson (1995) tentou corrigir alguns problemas metodológicos dos procedimentos adotados por Alling e $\mathrm{Da} \mathrm{Cu}$ nha, estendendo suas descobertas para sujeitos humanos. Ele treinou seus participantes a clicar com um mouse em dois quadrados apresentados em uma tela de computador. Clicar no quadrado da esquerda sempre produzia um som em VR8. Na presença do som, clicar no quadrado da direita produzia pontos, mas somente na presença de um "fundo de tela" específico que era controlado por um esquema de tempo variável. Este autor argumentou que o "fundo de tela" funcionaria como uma OEC-T para a resposta de clicar no quadrado da esquerda, dada sua correlação com a efetividade reforçadora do som. Os resultados obtidos por Hixson (1995) foram consistentes com sua hipótese inicial. Respostas no quadrado esquerdo na ausência do fundo de tela específico (OEC-T) diminuíram de freqüência. Entretanto seus resultados também podem ser interpretados como encadeamento de respostas.

Miguel e Andery (1998) tentaram demonstrar o controle da operação estabelecedora condicionada transitiva sobre o comportamento de ratos. As sessões experimentais iniciaram-se com uma caixa operante escura, situação na qual respostas a uma barra localizada à direita do bebedouro produziam como consequiência o acendimento de uma luz . Na presença da luz, respostas à uma barra esquerda só produziam água como conseqüência quando um som estava em curso. $\mathrm{O}$ aparecimento do som foi controlado por um VT6s. que era acionado após o aparecimento da luz, permanecendo ligado durante $10 \mathrm{~s}$. Os autores supuseram que som não estaria correlacionado com a disponibilidade da luz como um reforço condicionado, mas como uma condição que estabeleceria sua efetividade (da luz). Numa fase de teste os sujeitos foram expostos a uma sessão de $30 \mathrm{~min}$. na qual, o som era apresentado isoladamente controlado por um esquema de VT6s. Caso o som, evocasse, nessa sessão de teste, respostas de pressão à barra direita, ele estaria funcionando como uma OEC-T. Nenhum dos sujeitos mostrou resultados consistentes com essa hipótese.

Um dos problemas do procedimento adotado por Miguel e Andery (1998) foi que o aparecimento do som (suposta OEC-T) foi controlado por esquema VT6s que era acionado sempre após o aparecimento da luz (suposto reforçador condicionado). Tal delineamento evitaria a interpretação do som como um estímulo discriminativo correlacionado com a disponibilidade da água no final da cadeia. Entretanto, esse procedimento permitiu que o som adquirisse funções discriminativa e reforçadora condicionada, antes exercidas pela luz. Uma característica importante do procedimento adotado por Miguel e Andery (1998) foi a utilização das sessões de teste em extinção, que permitiu(ria) a observação da função evocativa da OEC-T. 
Se tomarmos como base todos esses experimentos, a condição de estímulo que foi chamada de OEC ou $\mathrm{S}^{\mathrm{E}}$ poderia claramente ser confundida com um estímulo discriminativo. Mesmo a partir da definição de $S^{\mathrm{D}}$ apresentada por Michael (1982; 1993a; 1993b) ainda é possível interpretar a presença da suposta OEC como sinalizadora da disponibilidade do reforçador condicionado, juntamente com o primário, pelo menos em encadeamentos simples, em que respostas e conseqüências estão temporalmente muito próximas umas das outras.

\section{Operações Estabelecedoras na Análise Aplicada do Comportamento}

De acordo com a discussão aqui apresentada parece que o conceito de operações estabelecedoras, principalmente as condicionadas transitivas, carecem de demonstrações experimentais. De acordo com Michael (1993a):

Estas relações complexas podem não desempenhar papel fundamental em experimentos típicos com animais porque elas requerem histórias muito extensas. Entretanto elas poderiam ser esperadas em espécies que possuem uma longa história de vida, em seus ambientes naturais, e certamente, em humanos (p.205).

Apesar da falta de consistência na área de pesquisa básica com animais, o conceito de operações estabelecedoras, incluindo as condicionadas transitivas, vêm cada vez mais se difundindo na análise aplicada do comportamento (Agnew, 1998; Fisher, 1996; Hall \& Sundberg, 1987; McGill, 1999; Smith \& Iwata, 1997; Vollmer \& Iwata, 1991; Wilder \& Carr, 1998).

Smith e Iwata (1997) defendem a identificação de operações estabelecedoras no diagnóstico e tratamento de distúrbios comportamentais. De acordo com os autores, uma análise funcional não deve somente incluir a identificação da contingência entre comportamento e conseqüência, mas também as condições que estabelecem a forma de reforçamento que mantém o comportamento, até porque a apresentação de tais condições seria responsável pela emissão da resposta (controle evocativo). Por exemplo, Durand e Crimmins (1988) e O'Reilly (1999) sugeriram que comportamentos-problema mantidos por atenção têm maior probabilidade de ocorrer em ambientes pobres em contatos sociais. Em casos desse tipo, a manipulação sistemática de condições estabelecedoras (o aumento nas interações sociais) poderia ser suficiente para a redução do comportamento problema.

Outro exemplo de manipulação de OEs é o estudo conduzido por Cameron, Ainsleigh e Bird (1992). Estes reduziram comportamentos agressivos mantidos por reforçamento negativo através da manipulação da tarefa que supostamente evocava tais comportamentos. Agressão ocorria sistematicamente quando o sujeito recebia um sabonete em barra e era solicitado a se lavar. Quando o experimentador passou a prover sabonete líquido, os comportamentos agressivos diminuíram. De acordo com os autores, segurar o sabonete aumentava o grau de dificuldade da tarefa estabelecendo a ocasião para que comportamentos que produ- zam a eliminação da tarefa (fuga) fossem reforçados.

McGill (1999) conclui que muitos dos procedimentos utilizados na área de análise aplicada têm como mecanismo comportamental a manipulação de variáveis motivacionais ou maniplulação de OEs. Um exemplo seria procedimentos que envolvem o que é chamado de "momento comportamental" 14 para aumento no comportamento de seguir regras (ou obediência ${ }^{15}$ ), em que sequiências de comandos prováveis de serem seguidos pelo sujeito (high-p) são apresentados imediatamente antes de comandos que tipicamente não são seguidos $(l o w-p)$. Tal procedimento resulta em 1) aumento na probabilidade de seguimento de comandos $l o w-p$ e 2) diminuição de comportamentos problema tipicamente mantidos por reforçamento negativo (fuga) (Mace \& Belfiore, 1990). De acordo com McGill (1999), a apresentação de comandos prováveis de serem seguidos (high-p) funciona como uma operação estabelecedora condicionada reflexiva inversa (OEC-R) por estabelecer sua própria redução como uma forma de punição, além de que o pareamento desses comandos com os comandos low- $p$ acaba por produzir transferência das propriedades motivacionais. Nesse caso, os comandos low- $p$ funcionariam como uma operação estabelecedora substituta (OEC-S). McGill, também interpreta outros procedimentos usados por analistas do comportamento como envolvendo manipulação de OEs.

Wilder e Carr (1998) afirmam que a manipulação de operações estabelecedoras parece ser o mecanismo responsável pela diminuição na frequiência de respostas em procedimentos de redução de comportamentos aberrantes como os de reforçamento não contingente (RNC) (Marcus \& Vollmer, 1996). Nesse procedimento, após uma análise funcional experimental (Iwata, Dorsey, Slifer, Bauman \& Richman, 1994) a conseqüência identificada como responsável pela manutenção do comportamento é liberada ao sujeito em esquemas de tempo fixo (FT) ou variável (VT). Tal procedimento tem se mostrado eficaz na redução do comportamento-alvo, provavelmente por conta de um mecanismo análogo ao da saciação. Estudos mais recentes (ex. Khang, Iwata, Thompson \& Hanley, 2000; Lalli, Casey \& Kates, 1997) apontam para o mecanismo de extinção como um dos responsáveis pela redução do comportamento-alvo, já que é comum durante procedimentos de reforçamento não contingente, que tal comportamento deixe de produzir suas conseqüências mantenedoras (extinção). Entretanto, em alguns casos (Hagopian, Crockett, van Stone, DeLeon \& Bowman, 2000) o procedimento de reforçamento não contingente pareceu produzir redução no comportamento-alvo mesmo quando tal comportamento continuou produzindo suas conseqüências mantenedoras (sem extinção), pelo menos em esquemas densos (conseqüências são liberadas mais freqüentemente), apontando novamente para a saciação $(\mathrm{OE})$ como um dos possíveis mecanismos responsáveis pela redução comportamental.

14 Behavioral Momentum

15 Compliance 
$\mathrm{Na}$ área de comportamento verbal, o conceito de operações estabelecedoras vem sendo largamente usado no treinamento de mandos. A própria definição de mando proposta por Skinner ${ }^{16}$ foi revista após a publicação do artigo de Michael $(1988)^{17}$ para incluir o termo operação estabelecedora como principal variável de controle.

Hall e Sundberg (1987) desenvolveram um procedimento chamado "cadeia interrompida" para treinamento de mandos. Nesse procedimento, sujeitos são treinados a desempenhar uma seqüência de atividades como, por exemplo, preparar sopa instantânea usando uma panela, água quente e uma colher, sendo que no final o sujeito acaba por consumir a sopa (reforço). Em um outro ambiente, os sujeitos aprendem a nomear os objetos usados (tato). Quando novamente são solicitados a desempenhar a cadeia comportamental (preparação da sopa), os experimentadores retiram um dos componentes necessários para a execução da cadeia (manipulação de operações estabelecedoras), na tentativa de 1) aumentar a efetividade desse componente como reforçador (função estabelecedora) e 2) evocar o comportamento (mando) que produz a obtenção deste componente (função evocativa). Tal procedimento vem sendo largamente adotado para treinamento de mandos em crianças com distúrbios de comunicação (para uma revisão da área de treinamento de mandos vide Shafer, 1994).

Parece que, independente de demonstrações experimentais consistentes, o conceito de operação estabelecedora foi definitivamente adotado por analistas do comportamento na tentativa de explicar tanto a ocorrência de comportamentos quanto a efetividade das consequiências que os mantém.

\section{Conclusão}

Entre as razões pelas quais acredito que o conceito de operação estabelecedora merece a atenção dos analistas do comportamento estão 1) porque tal conceito pressupõe relações ambientais que poderiam alterar o valor de reforçadores condicionados, independente do valor de reforçadores primários com os quais foram pareados, o que facilitaria o controle sobre respostas, que principalmente no caso humano, são quase que exclusivamente mantidas por reforçadores condicionados. 2) porque permite a modificação de comportamentos através da manipulação de eventos antecedentes, mantendo a relação resposta-conseqüência constante, 3) porque provoca uma nova discussão conceitual a respeito do uso do termo $\left.S^{\mathrm{D}} \mathrm{e}, 4\right)$ porque chama a atenção de analistas do comportamento para um dos mais importantes e tradicionais fenômenos psicológicos: a motivação.

16 O mando é um "operante verbal reforçado por uma consequência característica e portanto sob controle funcional de condições relevantes de privação ou estimulação aversiva... o mando especifica seu reforçamento" (Skinner, 1957/1992, p. 35-36)

17 O mando é um operante verbal cuja forma da resposta ou topografia é controlada por uma operação estabelecedora (Michael, 1988; 1999).
Como afirma Da Cunha (1995):

A proposta conceitual e metodológica de Operações Estabelecedoras, retomada por Michael, não apenas estabelece uma nova linha de pesquisa na análise experimental, como também prioriza a variável motivacional como evento ambiental tratado como uma variável independente, ou seja, enfatiza o estudo do controle desta variável sobre o comportamento (p.18).

Defende-se aqui, a necessidade de estudos mais aprofundados das variáveis ditas "motivacionais", não só por meio de novos delineamentos experimentais que tentem demonstrar esse controle, como também por meio de discussões teóricas que relacionem o conceito de operações estabelecedoras com a área de controle de estímulos (ex. discriminação condicional). Enquanto isso, parece coerente utilizarmonos do conceito de operações estabelecedoras, se acharmos que este pode contribuir para uma análise mais precisa do comportamento humano e de outros organismos.

\section{Referências}

Agnew, J. (1998). The establishing operation in the organizational behavior management. Journal of Organizational Behavior Management, 18 (1), 07-19.

Alling, K. (1991). The effects of a conditioned establishing operation on performance of a two-component chain. Dissertação de Mestrado. Western Michigan University.

Cameron, M.J., Ainsleigh, S.A. \& Bird, F.L. (1992). The acquisition of stimulus control of compliance and participation during an ADL routine. Behavior Residential Treatment, 7, 327340.

Catania, A.C. (1993). Coming to terms with establishing operations. The Behavior Analyst, 16, 219-224.

Cherpas, C. (1993). Do establishing operations alter reinforcer effectiveness?. The Behavior Analyst, 16, 347-349

Da Cunha, R.N. (1993). An experimental demonstration of the transitive conditioned establishing operation with pigeons. Tese de Doutorado. Western Michigan University, Kalamazoo.

Da Cunha, R.N. (1995). Motivação e análise do comportamento. Temas em Psicologia, 3, 11-18.

Durand, V.M. \& Crimmins, D.B. (1988). Identifying the variables maintaining self-injurious behavior. Journal of Autism and Developmental Disorders, 18, 99-117.

Fisher, W.W. (1996). The effects of establishing operations during functional analysis and treatment of aberrant behavior. Mesa redonda apresentada durante a 22 ${ }^{\mathrm{a}}$ Convenção anual da Association for Behavior Analysis, San Francisco, CA.

Hagopian, L.P., Crockett, J.L., van Stone, M., DeLeon, I.G., \& Bowman, L.G. (2000). Effects of noncontingent reinforcement on problem behavior and stimulus engagement: the role of satiation, extinction, and alternative reinforcement. Journal of Applied Behavior Analysis, 33, 433-449.

Hall, G. \& Sundberg, M.L. (1987). Teaching mands by manipulating conditioned establishing operations. The Analysis of Verbal Behavior, 5, 41-53.

Hesse, B.E. (1993). The establishing operations revisited. The Behavior Analyst. 16 (2), 215-217. 
Hixson, M.D. (1995). The development of a transitive conditioned establishing operation. Dissertação de mestrado. Western Michigan University, Kalamazoo.

Iwata, B.A., Dorsey, M.F., Slifer, K.J., Bauman, K.E. \& Richman, G.S. (1994). Toward a functional analysis of self injury. Journal of Applied Behavior Analysis, 27, 197-209.

Iwata, B.A., Smith, R.G. \& Michael, J. (2000). Current research on the influence of establishing operations on behavior in applied settings. Journal of Applied Behavior Analysis, 33, 411-418

Keller, F.S. \& Schoenfeld, W.N. (1996). Principles of psychology. B.F. Skinner Foundation. Copley Publishing Group. (Trabalho original publicado em 1950).

Kahng, S., Iwata, B.A., Thompson, R.H. \& Hanley, G.P. (2000). A method for identifying satiation versus extinction effects under noncontingent reinforcement schedules. Journal of Applied Behavior Analysis, 33, 419-431.

Knip, D.J. (1991). The effect of a conditioned establishing operations procedure on rats responding on a VI 30 schedule for sweetened condensed milk. Dissertação de mestrado. Western Michigan University, Kalamazoo.

Lalli, J.S., Casey, S.D., \& Kates, K. (1997). Noncontingent reinforcement as treatment for severe problem behavior: Some procedural variations. Journal of Applied Behavior Analysis, 30, 127-137.

Mace, F.C. \& Belfiore, P. (1990). Behavioral momentum in the treatment of escape-motivated stereotypy. Journal of Applied Behatior Analysis, 23, 507-514.

Malott, R.W. (1984). Rule-governed behavior, self-management and the developmentally disabled. Analysis and Intervention in Developmental Disabilities, 4, 199-209.

Malott, R.W., Malott, M.E. \& Trojan, E.A. (2000). Elementary principles of behavior. $4^{\text {th }}$ Edition. NJ: Prentice-Hall.

Marcus, B.A. \& Vollmer, T.R. (1996). Combining noncontingent reinforcement and differential reinforcement schedules as treatment for aberrant behavior. Journal of Applied Behavior Analysis, 29, 43-51.

McDevitt, M.A. \& Fantino, E. (1993). Establishing operations and the discriminative stimulus. The Behavior Analyst, 16 (2), 225-227.

McGill, P. (1999). Establishing operations: implications for the assessment, treatment and prevention of problem behavior. Journal of Applied Behavior Analysis, 32, 396-418.

McPherson, A. \& Osborne, J.G. (1986). The emergence of establishing stimulus control. The Psychological Record, 36, 375386.

McPherson \& Osborne, J.G. (1988). Control of behavior by an establishing stimulus. Journal of Experimental Analysis of Behavior, 49, 213-227.

Michael, J. (1982) Distinguishing Between Discriminative and Motivational Functions of Stimuli. Journal of Experimental Analysis of Behavior, 37, 149-155.

Michael, J. (1986). Repertoire-altering effects of remote contingencies. The Analysis of Verbal Behavior, 4, 10-18.

Michael, J. (1988). Establishing operations and the mand. The Analysis of Verbal Behavior, 6, 3-9.

Michael, J. (1993a). Establishing operations. The Behavior Analyst, 16 (2), 191-206.
Michael, J. (1993b). Concepts and principles of behavior Analysis. Kalamazoo: Society for the Advancement of Behavior Analysis.

Michael, J. (1999). EOs and the Mand. Material não publicado utilizado no curso 'Verbal Behavior', Western Michigan University.

Michael, J. (2000). Implications and refinements of the establishing operation concept. Journal of Applied Behavior Analysis, 33, 401-410.

Miguel, C.F. \& Andery, M.A. (1998, maio). An attempt to demonstrate the control of a transitive conditioned establishing operation in rats. Trabalho apresentado na $24^{\mathrm{a}}$. convenção anual da ABA: Association for Behavior Analysis, Orlando, FL.

Millenson, J.R. (1975). Principles of behavioral analysis. NY: Macmillan Co. (Trabalho original publicado em 1967)

Mineka, S. (1975). Some new perspectives on conditioned hunger. Journal of Experimental Psychology: Animal Behavior Processes, 104 (2), 134-148.

O'Reilly, M.F. (1999). Effects of presession attention on the frequency of attention-maintained behavior. Journal of Applied Behavior Analysis, 32, 371-374.

Schlinger, H.D. (1993). Establishing operations: another step toward a functional taxonomy of environmental events. The Behavior Analyst, 16, 207-209.

Shafer, E. (1994). A review of interventions to establish a mand repertoire. The Analysis of Verbal Behavior, 12, 53-66

Skinner, B.F. (1988). Ciência e comportamento humano. (J.C. Todorov e R. Azzi, Trad.) São Paulo: Ed. Martins Fontes. $7^{\mathrm{a}}$ edição. (Trabalho original publicado em 1953).

Skinner, B.F. (1991). The behavior of organisms. B.F. Skinner Foundation, Copley Publishing Group. (Trabalho original publicado em 1938).

Skinner, B.F. (1992). Verbal behavior. B.F. Skinner Foundation, Copley Publishing Group. (Trabalho original publicado em 1957).

Smith, R.G. \& Iwata, B.A. (1997). Antecedent influences on behavior disorders. Journal of Applied Behavior Analysis, 30, 342-375.

Sundberg, M. L. (1993). The application of establishing operations. The Behavior Analyst, 16, 211-214.

Vollmer, T.R. \& Iwata, B.A. (1991). Establishing operations and reinforcement effects. Journal of Applied Behavior Analysis, 24, 279-291.

Wilder, D.A. \& Carr, J.E. (1998). Recent advances in the modification of establishing operations to reduce aberrant behavior. Behavioral Interventions, 13, 43-59. 